Zagreb

AUTOUR DES STATUTA POLAE A. 1431

Quelques remarques et reinterprétations

O. Peu de statuts médiévaux de nos villes côtières nous renseignent aussi abondamment et en détail sur la pêche et les poissons que les Statuta Polae de $1431^{l}$. Le XLII ${ }^{\mathrm{e}}$ chapitre (De piscatoribus) énumère bien 46 espèces en les répartissant en quatre catégories et en en fixant le prix de vente. Cette répartition nous facilite l'identification des désignations qui aujourd'hui - ne sont pas aussi univoques comme on pourrait le conclure rien qu'en se basant sur les répertoires lexicographiques de la latinité médievale ${ }^{2}$. Or, il faut souligner d'entrée de jeu que les identifications et les valeurs de ces termes sont souvent négligées, fantaisistes et denuées de fondement. Et quand on les affuble de la désignation systématique en bonne et due forme, l'usageur du dictionnaire croit poux de bon avoir affaire à la vraie equivalence de l'ichtyonyme médiéval $^{3}$. Quand on sait combien ces sortes de "certitudes" lexicographiques ont la vie dure et combien vite elles font tache d'huile, la nécessité d'un sérieux réexamen de ces noms dans les dictionnaires de la latinité médiévale s'impose.

1. Après avoir decrete que le poisson frais ne peut être vendu nulle part nisi ad piscariam comunis, les statuts (abrégés SP) fixent les prix pour les quatre catégories de poissons séparé ment pour le temps de carême et pour le reste de l'année.

Contrairementà ce que $I^{\prime}$ on pourrait attendre, dans la catégorie des poissons les plus appréciés et recherchés, où la valeur économique a depuis longtemps nivele les noms, nous nous heurtons à quelques ichtyonymes peu clairs ou voire opaques. On y parle:

De barbonis

De varolis de grassa 


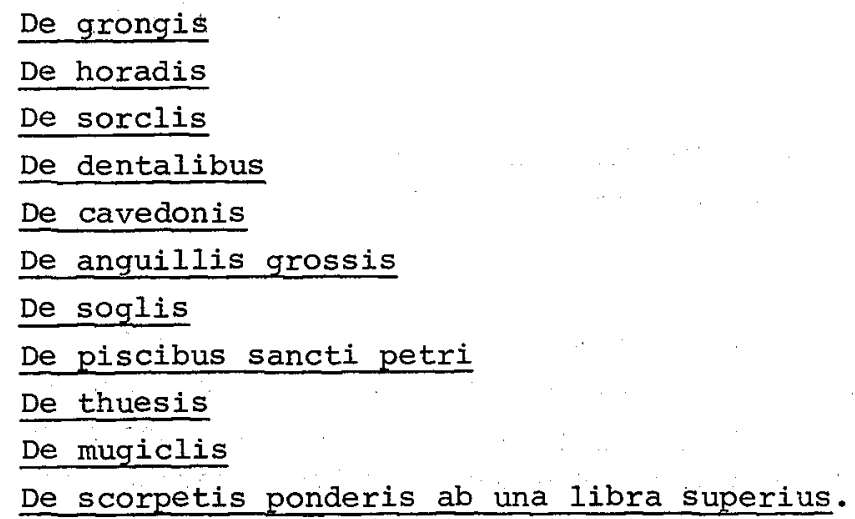

1.1. Dans de barbonis il s'agit évidemment de rouget de roche (Mullus surmuletus) connu depuis l'Antiquité pour l'excellence de sa chair. $\mathrm{s}^{4} 57 \mathrm{n}$ 'a donc pas raison de partir de barbo, -onis $n i$ de l'expliquer par "pesce barbo" qui est un poisson d'eau douce et en plus de qualité tout à fait négligeable. Il faut partir du vén. barbòn (latinise en barbonus) comme le fait LLMAJ ${ }^{5} 104$. 1.2. Sous de varolis de grassa se cache le nom du bar (Dicen= trarchus labrax = Labrax lupus) qui est un des poissons les plus recherches de 1'Adriatique, ou, pour être plus exact, sa variete D.punctatus. D'ailleurs, déjà Columelle $(8,17)$ distinguait les loups (=bars) tachetés de ceux qui ne le sont pas: tum etiam sine macula, nam sunt et varii. C'est de ces derniers que l'on parle dans le SP et l'etymologie de varolus est dans le qualificatif varius "bunt" REW 9157. Encore aujourd'hui en Italie, à Ancône, le jeune bar est appelé varolo, varoloto; "venez: variolo, varolo, ancon. varòlo lupo di mare giovane, coperto di macchiette" (A. Prati, Voc.etim.ital., I026). II s'ensuit que l'explication "muggine" donnée par s 609 ne tient pas debout et que celle de LLMAJ 1237 'labrax punctatus' est tout à fait exacte. 1.3. L'explication pour de horadis ne devrait pas poser de problème. Il s'agit de la daurade (Chrysophrys aurata) appelee de nos jours orada en vénitien et $(>)$ en croate comme il est noté en LLMAJ 543. On se demande pourquoi s 391 donne comme entrée (nom.sg.) oradis au lieu de orada? 
1.4. De grongis ( =Conger vulgaris), de dentalibus ( =Dentex vulgaris), de anguillis grossis ( =Anguilla anguilla; stade dans la croissance et la saison de la capture!), de soglis (=Soleidés et Pleuronectidés, d'après la forme populaire it. sogli/ol/a) ${ }^{6}$, de piscibus sancti petri (=zeus faber), de mugiclis ( =Mugil sp. et le-c- n'y est qu'un hypercorrectisme) et de scorpetis ponderis ab una libra superius ( =Scorpaena scrofa, les grands exemplaires de S.porcus; à l'exclusion de $S$. notata!) ${ }^{7}$ sont notés et expliqués correctement.

1.5. En revanche, le problème reste posé quant à l'expli= cation de trois espèces que les SP notent par de sorclis, de cavedonis et de thuesis. Voyons, pour commencer, comment nos deux répertoires expliquent ces trois ichtyonymes.

1.6. Sorclus (des SP) est explique par S 538 comme "pesce topo", tandis que LLMAJ 1104 explique vaguement "piscis genus" ce qui est encore moins clair quoique plus prudent. La question se complique par le fait que parmi les poissons de la II $\mathrm{I}^{\mathrm{e}}$ catégorie, donc moins chers, nous lisons de sorlis. S'agit-il de la même espèce mais de dimensions différentes? LLMAJ 1104 explique ce dernier par Trachurus trachurus c'est-à-dire "chinchard" ou "saureI commun" tandis que S 538 fait suivre sorlus d'un contradictoire et même impossible "sgombro bastardo, pesce della specie degli storioni" (!2) Il est clair que $\mathrm{s}$ a cru voir dans sorclus, que seulement -ć- oppose à sorlus, un déminutif de sorex (REW 8098; cf. en outre *soricare, *soricarius, *soricius, 8099-8101) mais, malheureusement, dans les nomenclatures ichtyonymiques mediterra= néennes nous ne trouvons aucun poisson recherché exprimant le sémantisme "souris"/"rat". Par contre, sorlus s'explique aisément comme déminutif de oaũoos / saurus que déjà Pline l'Ancien (NH 32, 151) note comme sorus à côté de saurus (NH 32,89$)^{8}$. Sur, šuro sont les formes encore vivantes en Croatie ainsi que le sont sur en triestin et suro en venitien. Dans tous ces ichtyonymes nous assistons à la convergence entre deux etyma devenus homophones: saurus "bunt", REW 7627 et subex (*sōber) "liège"; REW 8357, FEW 12,332. Comme il n'est pas permis de penser que de sorclis ( ${ }^{\text {ère }}$ catégorie) et de sorlis ( $I^{e}$ catégorie) désignent le même poisson, car, même 
s'il Etait de dimensions très grandes, le saurel ne saurait être un poisson recherche, il nous faudra laisser sans solution le problème de $\left(^{*}\right)$ sorclus en attendant une varia lectio qui pourrait nous renseigner davantage. En tout cas, ni "pesce topo" de $\mathrm{S}$, ni "piscis genus" de LLMAJ ne nous disent rien. 1.7. Toujours dans la I ère catégorie nous lisons de cavedonis et l'explication que $s$ et LLMAJ en donnent est tout à fait inverse de celle du cas précédent. C'est $S$ (141) qui reste circonspect et se contente d'un simple et on ne peut plus vague "pesce", tandis que LLMAJ 199 donne dans le piège de la ressemblance avec I'it. cavedine et déclare péremptoirement "piscis genus, cobitis barbatula" (sic!) ne tenant aucunement compte du fait que cobitis barbatula est un poisson d'eau douce qu'il est impensable de trouver à la poissonnerie de pola et encore moins parmi les poissons les plus appréciés. Mais ce n'est pas tout. De cavedonis ainsi "expliqué", on renvoie à chadelus qui est explique par le croate brkica! Les causes de ce renvoi pour le moins curieux sont a chercher chez $\mathrm{S}$ qui à la p.96 explique cadelus par "cavedine, pesce". Les redacteurs du LLMAJ n'ont fait que le suivre dans son explication exronnée. Mais, cette fois-ci la faute est beaucoup plus sérieuse car elle pouvait être facilement évitée dans: les deux répertoires si 1 'on avait pris en consideration que chadelus apparait par deux fois dans le même SP: une fois dans. la IV catégorie, donc parmi les poissons qui devaient etre vendus au plus bas prix, et une seconde fois quelques pages plus loin où le statut défend de débiter les poissons dépecés: non devastando tamen seu incidendo aliquos pisces exceptuatis ragiis, ch: a d e I is, squadris et piscibus bo. Par conséquent, il est évident que chadelus est un poisson cartilagineux, au même titre que la raie (Raia), la roussette (Scyliorhynus), les anges de mer (Squatina) et les deux pocheteaux (Raia oxyrhynchus/R.macrorhynchus) parmi lesquels il figure dûment dans la $I^{e}$ catégorie ${ }^{9}$. Mais ce qui pouvait en premier lieu faire eviter la bévue c'est le fait que cadel est vivant de nos jours tant parmi les pêcheurs italophones de la région. (Rovignoet Trieste: cadel) que chez les pêcheurs croates (kadeja et kadić) où ces formes continuent. le lat. catellus "junger Hund", REW 1763 et FEW 2/1, 498. 
1.8. Il nous reste à apporter un peu plus de clarté dans le de cavedonss qui, de toute évidence, au moyen âge denotait plusieurs referents. Nous croyons avoir montre plus haut qu'il n'y peut être question $n i$ de Cobitis barbatula ni d'un Mugilide. Mais, si le contenu ne correspond pas à la rélité dans les deux glossaires, 1'etymon présupposé est au contraire exact. Cavedonus dans les SP continue le lat. capito, -ōne REW 1638, FEW 2/1,264. Le sens y est "à grosse tête" et dans notre cas ce n'est ni la chevesne ni le muge (Mugil capito) ${ }^{10}$ mais au contraire une varieté d'anguille ou bien un stade bien caractéristique dans sa croissance. En effet, dans les SP, de cavedonis precède immédiatement de anguillis grossis. Il est connu que les anguilles peuvent différer par la forme de leur tête et les ichtyologues: $\mathrm{w}$. Luther et $\mathrm{K}$. Fiedler (1965, p.79) décrivent "les 'têtes larges' [qui] sont carnassières et les 'têtes pointues' [qui] vivent de Crustacés, de vers et d'autres Invertébrés". Le sème "grosse tête" est exploité Evidemment dans les formes croates glaveš, pujoglavica, punoglavica, punjeglavica... où le lexène de base est toujours glava "tête". Pour notre propos il est beaucoup plus intéressant de noter qu'il en est de même en Italie ${ }^{11}$ : dans les vénéties nous trouvons testone, dans les Pouilles capomazzo, à Bari capemazze, capetone et, dans le Latium, la même anguille est appelée capitone. Tout ceci nous en dit long sur la pertinence du sème "grosse tête" et de son expression capitōne $>$ cavedonus $^{12}$.

1.9. 'La dernière forme qui n'est pas claire parmi les noms de poissons de la Ière catégorie est le problématique thuesus/de thuesis. S (597) aussi bien que LLMAJ (1190) ne peuvent nous dire que ce que nous savions - qu'il s'agit d'un poisson. Etant donne qu'aucune forme semblable ne figure dans la diachronie de taxonomies mediterranéennes que nous connaissons, que $t(h)$ uesus ne se rencontre même pas comme appellatif et qu'il nous a été impossible de trouver une forme même vaguement ressemblante qui puisse constituer le point de départ formel pour un nom de poisson, nous avons tout de suite pensé a la possibilité d'une lecture défectueuse ou à une inadvertance du copieur. Nous avons alors 
changé de procédé et commencé à chercher quelles étaient les espèces de bonne qualité dont le prix de barème faisait defaut dans les SP. C'est ainsi que nous avons constate l'absence du thon rouge (Thunnus thynnus) et cela d'autant plus que le prix d'un autre Thunnide, le bonite à dos raye (Sarda sarda) figurait parmi les espèces de la II $^{e}$ catégorie sous une forme quelque peu insolite - de pallamilis ${ }^{13}$. Ia constitution trisyllabique du mot et surtout le th- initial nous engageait sur cette voie. Un obstacle de quelque poids pouvait constituer la rarete du thon le long des côtes istriennes. Mais, à ce propos nous connaissons les constatations auxquelles était parvenu petar Lorini, le plus grand spécialíste pour les questions des pêcheries en Adriatique. Son opinion sur la presence du thon dans les eaux de l'Istrie est ainsi rapportée par un expert italien: "Il direttore della societa Austriaca di pesca e di piscicultura marina, cav. Pietro Lorini, nel Manualetto del pescatore (... Trieste, 1914) in un articolo sulla pesca del tonno, scrisse quanto segue: Lungo le coste della provincia di Trieste e di Gorizia pescasi pochissimo tonno ed in condizioni consimili si trova la costa occidentale istriana, lungo la quale si catturano qua e là piccole partite di tonno, ma mancano impianti speciali per questa pesca. Il tonno non frequenta $i$ bassi fondali di detta costa, ma si presenta invece in masse lungo quella orientale istriana che ha acque profonde.. va notato che $i$ branchi minori possono passare pei canali interni di dette isole, parallele al continente, mentre $i$ più grossi passano al largo di esso... I pochi tonni che superano $i$ bassi fondi di Promontore, tenendosi al largo, penetrano nel golfo triestino" 14 . L'unique objection qu'on pourrait faire à la conclusion que le thuesus des SP est le thon résiderait dans les dimensions de ce poisson. Etant donné que cette espèce peut atteindre le poids de plus de $100 \mathrm{~kg}$, il serait logique que le statut le rangeât parmi les poissons quos [piscatores] possint incidere pro vendendo si voluerint. Mais, ce qui pour nous a fait pencher la balance du côté du thon se trouve dans un arrêté de Giustin Donà per la Serenissima Republica di Venezia, conte e proveditor di pola e sua giuxisdizione daté du 19 octobre 1717. On y rétablit les 
vieilles règles concernant la vente des produits alimentaires perche dunque cessi cosi una volta si danato libertinaggic di vendere senza stima li viveri... Le premier paragraphe parle des poisson= neries du marché de pola et on y fait mention des anciens statuts (Che non caminando più $i$ tempi felici nei quali furono stabiliti. li statuti e provvedimenti nè più le passate beneditioni massime nelle pesche...) et, ce qui nous intéresse le plus, on classe les poissons en quatre catégories. Pour la première lesiespèces sont énumérées nommément: ... e debbano li pescatori contenersi con la norma seguente consona alla convenienza ed al giusto: cioè $1 i$ barboni, varioli, orade, dentali, sfogli, scarpene, $t \circ n i$, palamide ed altri pesci d'una libra in su tutto I'anno possino venderli a soldi cinque; 1 'altro inferiore ${ }^{15}$ quattro, la minutaglia ${ }^{16}$ tre... Comme on le voit facilement, neuf espèces des SP de 1431 sont reprises dans le même ordre en 1717. On ne parle plus de grongis, de sorclis, de cavedonis, de anguillis grossis, de piscibus sancti petri ni de mugiclis. Si l arrêtê de Giustin Dona avait mentionné encore la doré (=piscis sancti petri= Zeus faber), on aurait pu dire tranquillement que les temps et les goûts ont change et que les poissons en sont venus à se classer dans les catégories qui sont valides pour les poisonneries de nos jours: ${ }^{17}$. En tout cas, de 1 examen de ces deux listes de prix il ressort clairement que varolus de grassa est le bar et que 1 'insolite et Etrange thuesusn'est autre chose que le thunnus.

2. Jusqu'ici nous avons traite des poissons recherchés et nous savons bien que la valeur Économique nivèle les noms. Plus nous descendons dans la qualité et le prix, plus variés seront les noms et moins vastes les aires quilils recouvrent. Pour cette raison, noús pouvons dire que les poissons classés dans la deuxième catégorie sont désignés par des noms dont l'expression s'est conservée dans les ichtyonymes modernes. Bien entendu, il n'en sera pas de même pour les poissons de la III $^{e}$ catégorie et pour ce que I'arrêté de Giustin Donà appelle la minutaglia. D une maniere génerale, on peut dire que nos deux glossaires ont cors: rectement interprété les noms en question. Nous donnons les noms systématiques en ne nous arrêtant que sur les interprétations où 
stimpose un peu plus de précision.

2.1. Item libra de infrascriptis piscibus debeat vendi per piscatores toto tempore anni pro soldo uno, et tempore quadragesime per denarios quatuordecim videlicet:

$\frac{\text { De merlis }}{\text { De lepis }}$
$\frac{\text { De corvelis }}{\text { De umbrelis }}$ (=Corvina)
De cevalis

De pallamilis

De scombris

De ragnis piscibus (=Trachinus)

\author{
De sorlis \\ De ribonis : (Pagellus erytrinus) \\ De baragiis \\ De scorpenelis \\ De piscibus bocha in chavo \\ (=Uranoscopus) \\ De ocladis et lasertis \\ De pighis \\ De agis (=Belone)
}

2.1.1... de me $r l$ i s. A notre avis, il s.agit du merle (Labrus merula) et non du merlu (Merluccius merluccius) comme le voudrait le LLMAJ 724 ou du serran Ecriture (Serranus scriba) comme nous lisons dans S 362. Il est bien connu que les dialectes italiens désignent le merlu par un continuateur de merlutius ${ }^{18}>$ it. merluzzo ce qui est originairement le nom de la morue (Gadus morhua) et $c^{\prime}$ 'est ce qui a induit en erreur les rédacteurs du LLMAJ. Au vén. merluzzo (Boerio 413) sont empruntées les formes croates merluc, marluc, morluc, murluc et var. En revanche, les noms du merle (it. tordo nero) continuent le lat. merula qui vit de nos jours en Dalmatie sous la forme merla, mijerla, mjerla ${ }^{19}$. A Bari ce même poisson s'appelle mèrrue. A cause de ces continuateurs, il nous semble plus justifie de voir dans de merlis des SP I'ablatif de merla et non de merlus.

2.2. de $s$ c o m b i $s$ (Scomber scomber) ne figure pas dans le Vocabolario de $s$.

2.3. de 1 as e $r$ t $i \mathrm{~s}$. Les SP distinguent les deux scombridés: Scomber scomber et Sc. colias. Ce dernier réapparâ̂t dans la III ${ }^{e}$ catégorie parce qu'il est moins recherche et que 1 'on tient compte de ses dimensions. De l'autre côtê de l'Adriatique 1'ichtyonyme ( L lacerta REW 4821) est du genre masculin tanđis que de ce côté-ci c'est plutôt laserta qu ìl faudrait écrire.

2.4. de $p i g h i s$. Pour ce qui est des noms de ce Labride 
il $n^{\prime} y$ a pas de doute que le nom.sg. est piga. En effet, c'est uniquement dans la partie orientale de l'Adriatique que le gr. puxís/cúnns a donné pika, fika, filka, finka et - figa d'où le "faux" calque croate de smokva ("figue"), smokvelj (<-ellus), smokvača etc. Sur les côtes italiennes ces Labridés ne sont jamais désignés par les expressions au contenu "figue". Les formes it. figo, figa, figora désignent toujours phycis sp., c’est-à-dire la mostelle ${ }^{20}$ ou bien stromateus fiatola où il faut voir la dénomination métaphorique au contenu "pudendum muliebre". A part la forme du nom. sg., les deux glossaires donnent la valeur exacte, car Labrus bimaculatus (LLMAJ 851) = Labrus mixtus (S 434). 3. Les vraies difficultés de l'identification commencent avec les poissons de la III ${ }^{e}$ catégorie. A peine ici nous nous heurterons à des ichtyonymes pour lesquels nous ne pouvons envisager aucune solution.

Item guod libra de infrascriptis piscibus vendatur per dictos piscatores toto tempore anni pro denarios decem et tempore quadragesime pro soldo uno videlicet

\begin{tabular}{ll} 
De picudi & $\frac{\text { De lasertis }}{\text { De mostelis }}$ \\
De malpis & $\frac{\text { De brugis }}{\text { De canteris }}$ \\
\hline
\end{tabular}

3.1. de $p i c$ u di. Les auteurs des deux glossaires hésitent à préciser la valeur du nom. Pour s 434 c'est "forse Sargus annularis, pesce", c'est-à-dire le sparaillon (=Diplodus annularis), tandis que le LLMAJ $n$ "entre pas en conjectures et se contente $d$ "un "piscis genus" en optant pour le nominatif picudis (?). Pourtant, ici au moins, la solution était à portée de la main. Picudus est le nom du sar tambour que les nomenclatures officielles italiennes nomment sarago pizzuto (Charax/Puntazzo/ puntazzo). La forme la plus proche est le triestin pissudo/pisso d'où le croate pišud/pis. Les formes istriennes sont attestées par P. Gilles (P. Gylilus). Dans le chapitre De melanderinis (De naminibus...p.556) il décrit le poisson en ces termes: "Pisces sargis aut melanuris similes excepto rostro quod acutius est, parvis dentibus non serratis, sed hominis similibus, optimi cibi. 
Ex Istrias Venetias afferuntur, picij vulgo nuncupati". Chez nous, déjà Petar Hektorović le mentionne dans son Ribanje (v.1117), mais en dépit de tout cela, au Moyen âge les Istriens le tenaient en faible estime.

3.2. de $\mathrm{s}$ a $1 \mathrm{p}$ i $\mathrm{s}$. Impossible de se méprendre quant au nom de la saupe (Box salpa = Boops salpa) car "Salpa ubique gentium nomen suum retinet", comme le constatait déjà au XVI ${ }^{e}$ siècle $P$. Gilles (De nominibus... 557). C'estpeut-être pour cette raison que $S$ ne croit même pas devoiir en faire mention dans son Vocabolario. 3.3. de $\mathrm{m}$ a $1 \circ \mathrm{d} i \mathrm{~s} i \mathrm{~s}$. Disons d'emblée que nous ne savons absolument rien sur la valeur de cet ichtyonyme. Il s'agit d"un ostéichthyen, et c'est hors de doute car le SP ne mélange pas les poissons osseux, les cartilagineux et les mollusques. Ces derniers figurent comme il se doit à part (v. plus bas 5 ). Dès lors, 1 'explication de $\mathrm{S} 342$ "pesce o mollusco"2l n-a aucun fondement non plus que celle de LLMAJ 688 qui le suit litteralement (piscium vel molluscorum species). Il est vain de se perdre en suppositions, car aucune nomenclature méditerranénne ne présente de forme qui puisse être rapprochée de celle-ci. Tout ce que nous pouvons conjecturer c"est qu'il s'agit d un nom frappe d"interdiction (le lexème malo- ?) mais pour en être sûr il nous faudrait connâ̂tre la valeur du nom ou du poisson en question, les habitudes et les croyances locales de Pola au XIV $\mathrm{XI}^{\mathrm{e}}$ siècle etco, etc. Nous avons essaye avec les noms des espèces soumises habituellement au tabou

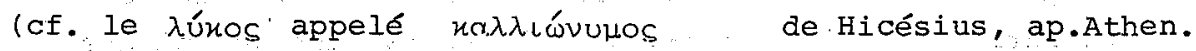
7,282d). De ce côté-ci de I'Adriatique c'est à coup sûr le cas pour la mostelle (Gaidropsarus = onos mediterraneus), mais, comme on le verra plus bas 3.5 , ce $n$ 'est pas le malodisus. Un autre poisson méditerranéen redouté à cause de ses piquants venimeux, Spánalva et soanalvís des Grecs, la vive (< vipera), c'est-a-dire Trachinus draco et $\mathrm{Tr}$.vipera, est bien range à sa place sous de ragnis piscibus. La murène (Muraena helena) dont abondent les légendes médiévales n'apparâ̂t pas dans 1 'Adriatique septentrional tandis que 1'oủpavoruóros d'Aristote (que

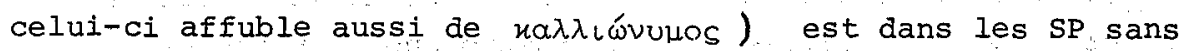
aucun doute appelé par la même lexie que de nos jours (piscis 
boc $(h)$ a in $c(h)$ avo $(v .2 .1$.$) . Il ne nous reste que d'avouer notre$ ignorance et de dire, comme le faisait Meillet pour les cas sem= blables, "valeur et etymologie inconnues".

3.4. de 1 a se $r$ t $s$ v. plus haut 2.3.

3.5. de $m \circ s t e l i s$. Tout de suite $i l$ faut rejeter

1 'explication fantaisiste et contradictoire du LLMAJ 746 "piscis genus, conger": parce que le congre (Conger vulgaris) est rangé dans la $1^{\text {ere }}$ catégorie: de grongis. Mais, il y a pire: dans I'explication serbo-croate on lit "vrsta ribe, pas čukov, ugor", c'est-a-dire "espece de poisson, requin, congre". Qu'est-ce qu'est venu faire le requin avec le congre? En fait, ce n'est qu'une maus ? vaise lecture de S 374 ou nous lisons: mustelus "pesce, Mustelus leavis". Trompé par le nom systematique Mustelus laevis Risso 1826 (aujourd'hui Mustelus mustelus L.), sella a cru que mustelus des SP et Mustelus des systematiciens ne faisaient qu'un et, sans saviir ce qu'était Mustelus de Risso, a expliqué par la valeur "sélacien", "squale". ou, plus précisément, "Émissole", le terme trouve dans le $\mathrm{SP}^{22}$. Encore une fois la faute est à chercher dans le genre grammatical qu“il n'est pas possible de distinguer dans les ablatifs des substantifs latins des première et deuxième conjugaisons. Si sella avait pris mustela au lieu de partir de mustelus, une bevue lui aurait été épargnée car il serait tombé - peut-être - sur le nom génerique Mustela (première partie du binome systématique Mustela fusca, Mustela vulgaris) ou sur le nom spécifique mustella (Onos mustella Risso 1826) et il serait bien tombé, car mostela des SP est en effet le plus proche parent du poisson que les naturalistes appelaient ${ }^{23}$ Mustela vilgaris, la faute n'aurait pas été aussi grave et on serait resté en famille - des Gadidae, bien entendu. De mustelis en SP se rapporte à mustela, nom que les Romains (Pline NH 9,63 et Columelle RR. $8,17,8)$ donnaient à un Gadide ${ }^{24}$ que nous appellons aujourd hui Phycis (bilennoides, Phycis phycis). Encore de nos jours dans les listes italiennes nous trouvons mustella de fundo (Phycis blennoides) et mustella de scheuggio (Phycis phycis). Carlo Battisti (DEI 2538) tiènt ces formes pour savantes quoiqu on les trouve en Ligurie et en Corse et même en Sardaigne ${ }^{25}$. En revanche, les formes populaires qui en sont issues sont bien 
nombreuses: musdea, musdeca et le sarde mustia, toujours comme noms de Phycis sp. L 'apparition de mustela dans les SP devra attenuer ce jugement.

Mais ce $n$ 'estipas à cause de $I$ 'inexactitude de $s$ (et de LLMAJ qui le suit) que nous nous sommes arrêté aussi longuement sur la valeur de la lexie de mustelis. C'était en premier lieu. pour démontrer le lien entre le scr. tabinja et le lat. tardif mustela et par là réponđre à la question que nous nous sommes posée plus haut (v. 3.3.). En nous assurant que mustela des SP désigne bien 1 espèce Phycis ( $\mathrm{Ph}$.phycis = $\mathrm{Ph}$.mediterranea; $\mathrm{Ph}$. blennoides), nous avons pu nous approcher sinon de l'etymologie au moins d'un facteur etiologique du nom croate tabinja (et var.) qui désigne les mêmes poissons. Or, ce dernier, quoique par la forme complètement different du mot latin, partage avec lui ce que $1^{\prime}$ on pourrait appeler le stimulus dénominatif. Tabinja est en effet le $\mathrm{s}$ e u 1 ichtyonyme serbocroate employe dans $t \circ u t e$ I'étendue de I'Adriatique orientale duquel nous ne savons absolument rien, car il est non seulement isolé et tout à fait opaque mais nous ne trouvons ni dans le fonds slave $\mathrm{ni}$ dans les nomenclatures mediterranéennes une expression quelconque qui puisse lui ressembler même vaguement. Les causes de cet isolement poux le moins curieux sont à chercher - à notre avis dans le caractère tabou qui entoure le nom de ce poisson. De même que le zonyme terrestre mustela "belette" a eté schon früh von der neuschöpfung eingeschränkt ${ }^{26}$ et à sa place nous trouvons dans toutes les langues des noms qui conceptualisent et expriment les idées de "belle", de "jeune mariée", de "amie" ou de "marraine"27, le zoonyme marin (transfert terrestria $\rightarrow$ marina) a suivi le même chemin: Phycis sp. est appelé à Bari jónola, à vasto (Molise) hulë putèllë, en Islande litla brosma, en bulgare galja (galja se "sich schmeicheln")et en turc gelincik balık (littér. "jeune mariee" + "poisson" et aussi "coquelicot") 28 . Un moment nous avons pensé que ce poisson à chair peu appréciée se cachait dans le de malodisis (v. 3.3.), mais puisque nous venons de voir que le nom latin mustela est bien clair dans les SP, il n'y a pas de doute que mostela désigne la phycis sp. (v. le francais savant 
mostelle) :

Il faut ajouter quelques mots sur I apparition de "conger" dans 1 'explication du LLMAJ. Nous avons déjà souligné la grande ressemblance entre les genres phycis et Motella ( =onos = Gaidro= psarus) qui appartiennent tous deux à la grande famille des Gadidae. Les deux poissons sont tellement ressemblants que sur une dizaine de points enquetés Motella, elle-aussi, porte le nom de tabinja ou bien sa variante masculine tabinjol (<-eolus) ou tabinjac. Qu'est-ce que nous pouvons conclure du reste des noms pour Motella sp.? Simplement, que de nom elle n'en a pas. On la désigne soit par le nom de tabinja qui recouvre 1 'ensemble \{Phycis + Motella\}, soit à l'aide d'un type dénominatif qui dénote sa prétendue parenté avec le congre. Autrement dit, le nom pour Motella sp. n'est pas prononcé (sauf si c'est tabinja) et s'il faut la nommer on dit que c'est "la mère du congre". La lexie prend alors la forme syntaxique romane mat od grunja (calque syntaxique d'après le veri: màre de grongo) ou la forme slave ugorova majka, grugova mati etc. Le contenu "mère" peut être remplace par "vieille femme" (gružja baba) ou "femme de la famille du congre" (grugovica). En dehors du croate et du venitien, on rencontre le même type de dénomination en Castille où la même espèce est appelée madre del congrio et légèrement modifie dans les Asturies et en Galicie: madre anguila. Ceci pourrait être en quelque sorte une des explications possibles pour 1 apparition du nom conger dans le LLMAJ •

Et, dernière hypothèse: si sur le marché au poisson de la ville de Pola on distinguait Phycis de Motella, il se pourrait que cette dernière fût appelée par le tout aussi éloquent malodisus. Mais, puisque les preuves nous font dëfaut, nous:ne faisons que nous perdre en conjectures...

3.6. de brugis. Ici encore nous somme dans I impossibilité de dire quoi que ce soit ${ }^{29}$. Le nom est isolé, opaque et inconnu. Au moins en ce qui nous concerne, aucune hypothèse ne s'offre à l'explication.

4. Il pesce mato ou, comme diraient les ichtyologues, les poissons cartilagineux sont rangés a part dans la $\mathrm{IV}^{\mathrm{e}}$ catégorie. 
Item guod libra de infrascriptis piscibus debeat vendi... per denarios sex......videlicet:

De ragiis (raia sp.)

De pardis

De chadelis

De squadris

De piscibus bo.

4.1. de pardis. Ici c'est le $\mathrm{s} 415$ qui commet la grosse erreur en expliquant par."Pagrus vulgaris, pesce" le prétendu pardus des SP! Un des poissons les plus appréciés de la Mediterranée, le pagre ( $g r$. páypos/ráyoos. , lat. pager, phager, pagrus, phagrus). le Pagrus pagrus ( =sparus pagrus) des systématiciens, aurait été vendu à Pola pour 8 deniers, tandis que les muges ou même les congres valaient deux fois plus. En rélite, le SP fixait ce prix: non pour le pagre mais pour la négligeable roussette (Scyliorhinus. stellaris) ${ }^{30}$ que les Italiens appellent et appelaient gattopardo $^{31}$, jattupardu, lobarda, que 1 'on mange seulement si $I^{\prime}$ on $\mathrm{n}^{\prime}$ a rien d'autre. Le croate de 1 'Adriatique superieure a conservé parda. C'est en effet la gapta petrosa des Statuts de la ville dalmate de Trogir (Trau) a.1372 ${ }^{32}$. A notre avis, c'est plutôt parda que párdus qu "il fallait écrire comme entrée.

4.2. de $c h$ a d elis. v. 1.7. 4.3. de s q u a driso. Cette fois-cis 547 part du féminin squadra et LIMAJ 1116 du masculin squatrus et cela parce que stat. Trag. a.1322 laisse distinguer la forme en usage: libra squatri vendatur duobos denariis. Au XVI ${ }^{e}$ siecle Konrad Gesner (Nomencl. p. 141) attestait le féminin pour les parlers italiens: "Graeci ólvn ... Itali squatina vel squadra...". Aujourd hui dans les Abruzzes squadrë m. (E. Giammarco, DAM 2097). Pour ce poisson (Squatina squatina $=S$. laevis) le croate $n$ 'a conserve que les formes provenant d'un déminutif * squatulus (Skok, 2 frPh 50, 526; $54,419)$ : šklatt, škl ${ }^{\circ} a t$, sklatina etc. tandis que les noms plus recents (škvajina, škvaja...) sont empruntés au ven. squaena (Boerio 697).

4.4. de $\mathrm{p}$ i $\mathrm{s}$ c i b u $\mathrm{s}$ b o. Manque dans le LLMAJ tandis que $S 439$ explique correctement mais pourtant sans précision 
" pesce bue". Il s'agit des deux très grandes raies (Raia/Dipturus/ batis = Raia macrorhynchus et Raia /Dipturus/ oxyrhynchus). Aucun doute quant à l'identification de ce poisson car on lit chez P. Gilles ( $P$. Gyllius) "Bos quem Plinius inter planos et cartilagineos commemorat ad trecentas aliquando libras accedit, Dalmatae etiam nostrae aetatis $\mathrm{b} \circ \mathrm{v}$ e m vocant" (De Nom.p. 561) ${ }^{33}$. Bien que opaque pour les locuteurs croatophones, le nom dalmate est encore vivant: buj et son augmentatif bujina ${ }^{34}$ ce qui se recouvre avec les formes siciliennes buju, vugghiu, bugghiu. 5. Les céphalopodes sont mentionnés à part dans un bref paragraphe:

Item quod sepe, luline e polpi vendantur ad occulum. 5.1. Ici encore, $S 336$ manque de précision quand il fait suivre lulina simplement de "mollusco". Il fallait préciser "calamaro. Loligo vulgaris". Mais, ce qui est clair pour les Dalmates ne l-était surement pas pour sella. qui y voyait un nom insolite que seulement le fait de se trouver entre sepe (seiche, sepia offi= cinalis) et polpi (pieuvre, Octopus vulgaris) designait comme un Céphalopode. En depit du très grand nombre d'attestationsdu zoonyme lol(l)igo dans le latin classique, cette forme s'est conservee uniquement dans le roman de Dalmatie gardant la valeur vellaire de $-g^{i}-(\text { lignja, oliganj, uligna ... })^{35}$. La forme istrienne que nous trouvons dans les SP est precieuse aussi à un autre titre. La consonne initiale de lol(1)igo, conque comme article defini, est partout tombée et ce n'est qu'ici que nous en trouvons une dernière trace.

6. Une manière de vente particulière est réservée pour les sauclets (Atherina sp.):

Item pisces minuti qui capiuntur cum $\operatorname{tracta}^{36}$ exceptis

a g u n c e $l$ i guod vendatur ad occulum.

Item quod centenarius de a g u n c e l is debeat

vendi per totum annum pro soldis duobus.

Pour les mendoles et les picarels Maena maena et Maena

smaxis) la vente est réglée avec la difference qui s'impose:

Item $\mathrm{m}$ e $\mathrm{n} 0 \mathrm{l}$ e debeat vendi toto tempore anni silicet triginta duo pro soldo uno et in quadragesima viginti menole pro uno soldo. 
6.1. Encore une fois Sella procède d'une manière négligé: aussi bien menolla de statut de Rab (Arbe) que menola de Umag (a. 1528) ou menula (sans indication de source) ${ }^{37}$ il traduit obsti= nément par "perca, pesce" ce qui n’a aucun sens, car menula/menola est d'usage courant dans les dialectes croates et italiens' (déminutif du lat. maena $=$ gr. uquís ) et désigne "le manger des pauvres" ${ }^{38}$. Elle est bien à sa place dans les SP pour clore la liste de la minutaglia.

\section{INDICATIONS BIBLIOGRAPHIQUES}

W. Luther - K. Fiedler, Guide de la faune sous-marine (traduction francaise de 1 'allemand "Die Unterwasserfauna der Mittelmeerküsten), Neuchâtel-Paris, 1965.

V. Valente, "Annotazioni al LLMAJ", Melanges Skok, Zagreb, 1985, 511 ss.

V. Vinja 1960, "Notes ichtyonymiques dalmates", SRAZ 9-10. 149-161. V. Vinja 1966, "Les noms des Ménidés. Essai d"étymologie globale", SRAZ, 21-22, 3-38.

V. Vinja 1l68-70, "Le roman de Dalmatie - intermédiaire des éléments grecs dans I 'ichtyonymie yougos lave", Bollettino dell ALM, 10-12,77-84 $\mathrm{V}_{2}$ Vinja 1976, "Poissons serpentiformes. Noms panméditerranéens $\sim$ noms à aire réduite", SRAz 4l-42, 195-226.

V. Vinja 1985, "Kirnja - un ichtyonyme typiquement dalmate", Melanges Skok, 527-538.

V. Vinja 1986, Etimologija i struktura naziva jadranske faune, I-II, Zagreb-Split.

Notes

1. Dott. B. Benussi, statuto del Comune di Pola, in "Atti e memorie della Società Istriana di Archeologia e Storia Patria", anno XXVIII - 1911, 108-449, Parenzo.

2. Le lexique médiéval de la latinité adriatique est plus parti= culièrement pris en considération dans les deux dictionnaires suivants: Pietro Sella, Glossario latino italiano (Stato della Chiesa - Veneto-Abruzzi) , Citta del Vaticano, 1944; Lexicon Latinitatis Medii Aevi Iugoslaviae, I-II, Zagreb, 1973-1978. 3. Dans un de nos travaux antérieurs (Vinja 1985) nous avons essaye de montrer par quelles voies le nom spécifique acharne (=cernier, Cerna et Polyprion) est venu à faire part du binôme Pagellus acarne qui designe le pageau (Pagellus).

4.= P. Sella, Vocabolario ...

5. = Lexicon Latinitatis Medii Aevi Jugoslaviae.

6. De soglis ne se trouve pas dans $\mathrm{S}$.

7. La valeur "pesce scorpione", donnée par $S 518$, est très approximative. LLMAJ 1059 se contente de tout aussi vague "piscis genus, scorpio". Dans les deux cas il fallait noter "scorpena". 
comme pour scorpenelus.

8. Cf. aurata et orata.

9. C'est il pesce mat(t)o pour les pêcheurs italiens, tandis que les pêcheurs croates disposent de 1 "archilexème divja ("sauvage") riba, luda ("matto") riba ou landovina, hljandrovina, kljandrovina... Pour 1.etymologie de ce dernier terme, v. Vinja 1986, 2.1.3., p. 50-51.

10. Le nom spécifique de capito (dans le binôme Mugil capito) a été introduit dans la systématique par Cuvier en 1829 et par Bonaparte en 1834 .

11. Pour les sources, v. Vinja 1976, 200.

12. Même les ichtyologues se servent de cette particularité pour leurs désignations systématiques: Anguilla var. macrocephala DE LA PYLAIE (1835), Anguilla latirostris JENYNS (1835) et même Anguilla capitone KAUP (1856).

13. LLMAJ 799 fait mention de pallamila et renvoie avec raison à palamita, palameta (797) od on lexplique par "pelamis (sic!), piscis genus". S 399, au contraire, a une entrée pallamila (?) expliquée correctement par "palamita, pesce".

14. Dott. Corrado Parona, "Il tonno e la sua pesca", Memoria LXVIII, $R$. Comitato talassografico italiano, Venezia, $\overline{1919,} \bar{p} .183$. 15. = II catégorie.

16. = III $\mathrm{e}^{\mathrm{C}}$ catégorie. Minutaglia traduit fidèlement de omnibus aliis piscibus minutis du SP. I arrêté de Giustin Donà est cité d'après B. Benussi, v. n. 1 .

17. Voir pour comparaison le relevé des prix pour la période de 1895 a 1904: S. Cihlár, III Pregled morskog ribarstva u Hrvatskom primorju od god. $1895 / 96$ do god. $1904 / 05$ (Senj, 1905).

18. V. dans le même S 362 merlutius, merlusus, a. 1309 ; cf. aussi DEI 1432 et Hugo Schuchardt, ZfrPh 30,724 .

19. Pour le synonyme mriljuj chez l'écrivain ragusain Marin Držic, v. Vinja 1986, p. 470 .

20. Pour plus de details, v. Vinja 1968-70, 80 ss.; cf. ici 3.5.

21. S donne malodiosus, tandis que LIMAJ suit la lecture de $B$. Benussi malodisus.

22. Mais, si avec cela nous savons pourquoi sella sétait-il trompe, nous ne savons pourquoi dans le LLMAJ on a introduit le congre qui n'a rien à voir ni avec mustelus ni avec le vrai semantisme du nom de poisson atteste dans les SP.

23. Nous soulignons appelaient car le nom générique Mustela $n$ 'est plus valide. On l avait remplace par Motella et ensuite par onos. Aujourd'hui, le nom valide est Gaidropsarus.

24. Mustela (=Motella = Onos = Gaidropsarus) des systématiciens est aussi un Gadidé.

25. Même en veste latine, de mustelis des SP parle éloquemment de la vitalite de la désignation.

26. "Das hängt damit zusammen, dass das wiesel im volksglauben als ein zauberwesen gilt, vor dem man sich fürchtet, daher scheut man sich seinen namen auszusprechen und ersetzt ihn durch ein wort, mit dem man dem tier schmeicheln möchte", Wartburg, FEW 6/3, 269. 27. Cf. A. Meillet, Linguistique historigue et linguistique générale, 1, 281; St. Ullmann, Précis de sémantique française, 1952,260 ss. et surtout la traduction espagnole de E. de Bustos 
Tovar, Madrid, 1965, p. 350.

28. Pour plus de détails, v. Vinja 1986, p. 264 ss.

29. S 86 "pesce", LLMAJ 136 "piscis genus".

30. Comme on 1 'a bien vu dans le LLMAJ 818 .

31. pardus, párdalos FEW 7, 641; REW 6231.

32. Insuffisamment expliqué par "piscis genus" dans le LLMAJ 500;

cf. jattupardu di scogliu en Sicile.

33. "Dalmatae etiam nostra aetate b o v e m vocant", Konrad Gesner, Nomencl. 132.

34. Cf. le vegliote bu chez M. Bartoli (Dalm. II, 175); V. Vinja $1960,154-5$.

35. REW n'a pas d'article dédié à cette forme, tandis que le FEW 5, 400 traite du lat. lolligo "tintenfisch" seulement à cause de 1.emprunt savant loliige "sépia" attesté en 1528. L“it. lolliqine est aussi une forme livresque (DEI 2265).

36. Conservé en dalmate trajta, trakta avec la même signification.

37. On ne fait pas mention des menola du SP.

38. Cf. prov. matasoldad "picarel" et le cat. madre soldad; en grec mod. rapisa signifie "picarel" et - "gens misereux", cf. Vinja, 1966,30 .

\section{SAŽETAK}

OKO STATUTA PULE IZ G. 1431

Prijedlog za neka nova tumačenja

Pulski statut iz 1431. godine, kako ga je pročitao i objavio B.

Benussi (a mi smo ga u svim njegovim čitanjima doslomo slijedili), pruža nam obilje podataka za srednjovjekovnu ihtionimiju tog dijela jadranske obale. Poučen iskustvom o upornom prenošenju iz rjeernikà u rječnik krivih interpretacija $i$ identifikacija naziva za morske organizme, autor ukazuje na nekoliko pogrešnih tumačenja srednjovjekovnih ihtionima koja proistječu iz nedovoljnog poznavanja referenata." Ukazuje se na kriva mjesta u djelu Pietra Salle i u Lexicon Latinitatis Medii Aevi Jugoslaviae, a istodobno se predlaže novo Čitanje za dosad neriješeno $t(h)$ uesus. U intionimu malodisus nazire se neko tabuom pogodeno ime, dok naziv sadržan u de brugis i dalje ostaje bez prihvatljiva rješenja. 